\title{
Motivos da adesão de idosos às Academias da Terceira Idade
}

\author{
Reasons for the adherence of older adults to Gyms for Seniors
}

\section{Elizabeth Rose Assumpção Harris' (ID Helder Guerra de Resende ${ }^{2}$ (D) Flávia Porto ${ }^{1 D}$ Nádia Souza Lima da Silva’ ${ }^{\mathbb{D}}$}

Palavras-chave:

Envelhecimento. Exercício Físico. Política Pública de Saúde. Saúde do Idoso.

Keywords: Aging. Physical Exercise. Public Health Policy. Health of the Elderly.

\footnotetext{
Universidade do Estado do Rio de Janeiro, Programa de Pós-Graduação em Ciências do Exercício e do Esporte, Rio de Janeiro, RJ, Brasil.

2 Universidade da Força Aérea, Programa de Pós-Graduação em Desempenho Humano Operacional. Rio de Janeiro, RJ, Brasil.
}

Financiamento da pesquisa: Coordenação de Aperfeiçoamento de Pessoal de Nível Superior - Brasil (CAPES) - Código de Financiamento 001. Bolsa de Mestrado.

Os autores declaram não haver conflito na concepção deste trabalho. 
like the teacher and It makes me feel good, both with 100\%) and their perception of the benefits of exercise (It is a healthy physical activity: 100\%). Conclusion: although health reasons are important for the older adults who enter the program, they mostly remain due to how much they like the activities.

\section{INTRODUÇÃO}

Com o aumento da população idosa no Brasil ${ }^{1}$, passou a ser cada vez mais evidente a necessidade de políticas públicas dirigidas aos mais velhos ${ }^{2}$. Para Harris et al. $^{2}$, alternativas têm surgido na forma de programas de assistência orientados aos idosos, inclusive relacionados à necessidade de estimular um estilo de vida ativo, uma vez que a prática regular de exercícios físicos (EF) vem sendo apontada como uma ferramenta importante para o controle de doenças crônicas, manutenção da autonomia funcional e da qualidade de vida de pessoas idosas ${ }^{3}$.

Por isso, torna-se cada vez mais comuns programas gratuitos de EF oferecidos em locais públicos, fornecendo apoio a populações que normalmente têm dificuldades para alcançar a meta mínima recomendada de práticas de atividade física, a exemplo da pessoa idosa ${ }^{2,3}$.

Uma alternativa de política pública baseada na prática gratuita de EF, no município do Rio de Janeiro (RJ), Brasil, é o projeto Rio Ar Livre (RAL), que possui como um de seus programas a Academia da Terceira Idade (ATI) ${ }^{4}$. As ATIs são academias compostas por equipamentos de ginástica instalados em locais públicos, que possibilitam a prática de EF orientada por profissionais de Educação Física ${ }^{5}$.

Considerando-se a importância da prática regular de EF para a saúde ${ }^{3,6}$, ressalta-se o valor desse programa para a promoção da saúde dessa população, especialmente por ser supervisionado e gratuito, o que, potencialmente, facilita o acesso de idosos que apresentam maior dificuldade para aderir a um programa dessa natureza ${ }^{7}$, fator primordial para que os benefícios dessa prática sejam sentidos ${ }^{2,3}$.

Levando-se em conta as características diferenciadas das ATIs, quando comparadas às de outros programas desenvolvidos em academias tradicionais, com ambientes e equipamentos mais sofisticados ${ }^{5}$, e seu status de política pública de promoção da saúde, torna-se relevante entender o que facilita a adesão da pessoa idosa a esse projeto, para que os motivos encontrados sejam usados como parâmetro para sua avaliação e reestruturação, a fim de aumentar sua qualidade e seu impacto.

Diante do exposto, o objetivo central deste estudo foi verificar os motivos de adesão de idosos às ATIs da cidade do Rio de Janeiro, assumindo que adesão compreende tanto ao seu ingresso quanto à sua permanência no programa.

\section{MÉTODO}

Este é um estudo transversal e descritivo, no formato de levantamento, pois visa descrever a distribuição das características que ocorrem naturalmente entre as pessoas idosas frequentadoras das ATIs da cidade do Rio de Janeiro.

As ATIs são academias vinculadas ao projeto RAL, dotadas de equipamentos de ginástica instalados em locais públicos, que possibilitam a prática gratuita de EF orientada por profissionais de Educação Física ${ }^{5}$. Embora não seja de uso exclusivo desse público, as ATIs atendem prioritariamente as pessoas idosas e possuem como exigência somente a liberação médica para a sua prática ${ }^{5}$.

A cidade do Rio de Janeiro é bastante heterogênea, apresentando diferentes graus de desenvolvimento. Para facilitar seu gerenciamento, diminuindo as desigualdades na distribuição e utilização de recursos disponíveis, o município foi dividido em cinco áreas de planejamento, que são subdivididas em 10 Áreas Programáticas (APs). Cada uma dessas áreas reúne bairros com características sociodemográficas similares ${ }^{8}$. Para garantir a representatividade dos diferentes perfis sociodemográficos, incluiu-se na amostra núcleos de academias de todas as APs da cidade. 
A quantidade de núcleos por APs foi determinada por calculadora de amostras ${ }^{9}$, totalizando 58 ATIs distribuídas por todas as APs do município (ano de referência: 2017). A determinação dos locais de coleta foi realizada por sorteio ${ }^{10}$, a partir de uma lista de endereços fornecida pela coordenação do RAL.

Adotou-se como critérios de inclusão estar inscrito e frequentando há pelo menos seis meses a ATI e possuir idade igual ou superior a 60 anos. $\mathrm{O}$ dimensionamento da amostra a partir dos sujeitos elegíveis foi realizado segundo Barrow e McGee ${ }^{11}$. Para ATIs com até 50 alunos foram incluídos $20 \%$ dos sujeitos com idade igual ou acima de 60 anos; nas ATIs com 50 a 100 alunos, 15\% e, finalmente, nas ATIs com mais de 100 alunos, 10\% dos frequentadores foram incluídos. Após sorteio, foram envolvidos 396 voluntários de ambos os sexos, com idades entre 60 e 91 anos. Todos os participantes assinaram o Termo de Consentimento Livre e Esclarecido (TCLE), conforme determinação da Resolução no 466/12 do Conselho Nacional de Saúde ${ }^{12}$. A pesquisa foi aprovada pelo Comitê de Ética do Hospital Universitário Pedro Ernesto (Parecer: 1.514.233).

Para levantar os motivos de adesão foi utilizado o questionário desenvolvido e validado por Castro ${ }^{13,14}$, que sofreu pequenas adaptações no texto de modo a adequá-lo ao público e à atividade física desenvolvida na ATI, pois a versão original tinha como foco diferentes populações e tipos de atividades.

O instrumento possui três partes que identificam: 1) o perfil socioeconômico do sujeito; 2) os motivos que justificam seu ingresso no programa; e 3) os motivos que justificam sua permanência no mesmo. Além disso, foram incluídas questões referentes à saúde dos investigados. $\mathrm{Na}$ parte que levantou os fatores de ingresso, os voluntários apontavam para cada item indicado se esse era o primeiro, o segundo, ou o terceiro principal motivo para o seu ingresso; enquanto na parte destinada aos aspectos relacionados à permanência indicavam o nível de importância de cada um, como muito importante, mais ou menos importante ou pouco importante.
Os questionários foram aplicados entre fevereiro e junho de 2017, nas próprias ATIs, nos horários de aula, por três pesquisadoras devidamente treinadas. Para evitar possíveis dificuldades por parte de alguns idosos, o instrumento foi preenchido pelas pesquisadoras.

A frequência das respostas foi determinada através do percentual. Para os motivos de permanência, levantados através de uma escala de opinião, níveis de $80 \%$ foram aceitos como acordo, percentual mínimo aceitável para considerar observações fiáveis, segundo orientações da fórmula de Bellack.

\section{RESULTADOS}

A Tabela 1 apresenta a distribuição dos participantes em cada uma das APs da cidade do Rio de Janeiro, bem como suas características quanto ao sexo e à faixa etária. Ressalta-se que na AP 5.3 houve somente uma pessoa entrevistada, por conta da quantidade de frequentadores que atendia aos critérios de inclusão. Assim, os dados dessa região foram acrescentados aos da região vizinha, a 5.2. A amostra total reflete o que ocorreu em todas as APs, ou seja, foi composta por maioria de mulheres e predominantemente por idosos na faixa etária mais baixa, apresentando uma redução gradativa da participação no projeto, conforme o avanço da idade.

Como não foram encontradas grandes variações entres as APs, optou-se em apresentar o perfil socioeconômico e da saúde da amostra total e comentar as poucas diferenças existentes na discussão dos resultados, assim foi feito também para os motivos de adesão dos sujeitos ao programa.

A Tabela 2 apresenta o perfil socioeconômico e indica que a maioria dos investigados possui baixa renda salarial, grande adesão ao projeto e histórico de prática regular de EF, especialmente a ginástica. Além disso, a maioria não depende de terceiros para frequentar o projeto e indica a proximidade da ATI com o local de moradia como um motivo bastante relevante. 


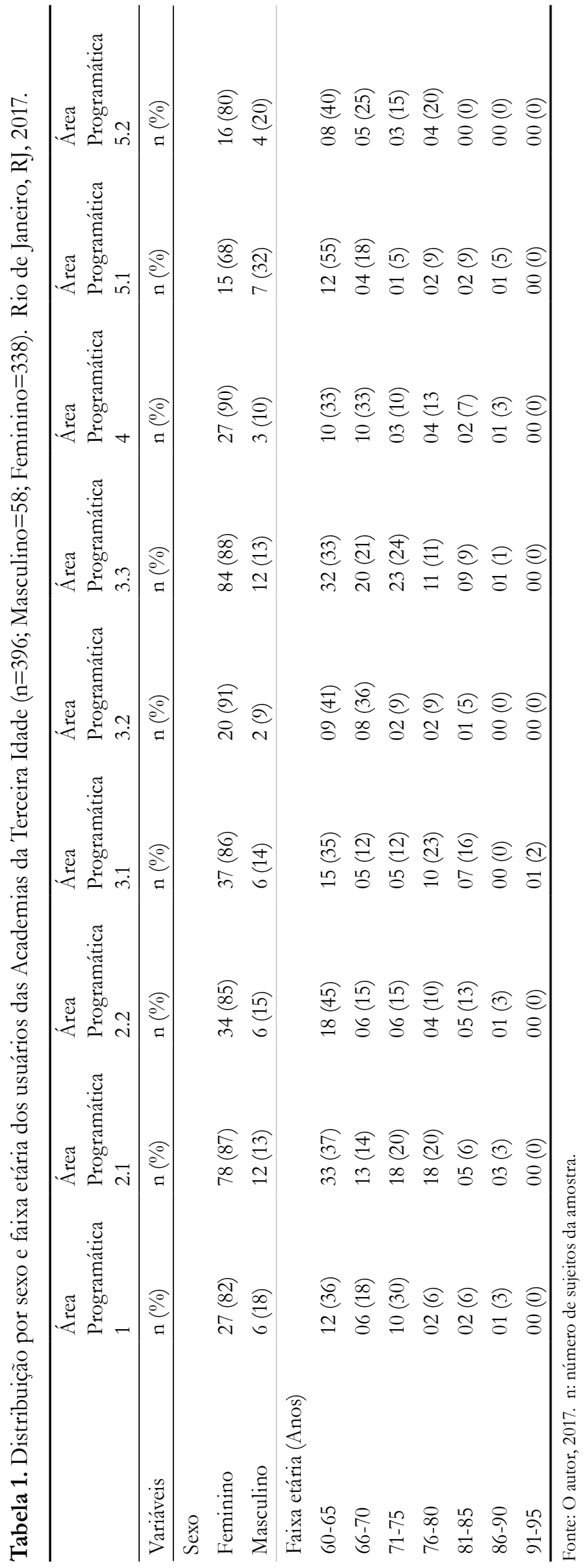


Tabela 2. Perfil socioeconômico e de saúde da amostra total. ( $n=396 ;$ Masculino= 58; Feminino= 338). Rio de Janeiro, RJ, 2017.

\begin{tabular}{|c|c|}
\hline Variáveis & $\mathrm{n}(\%)$ \\
\hline \multicolumn{2}{|c|}{ Renda pessoal (Salário mínimo) } \\
\hline$\leq 1$ & $249(62,9)$ \\
\hline $1-3$ & $145(36,6)$ \\
\hline $3-5$ & $49(12,4)$ \\
\hline$\geq 5$ & $34(8,6)$ \\
\hline Sem resposta & $26(6,6)$ \\
\hline \multicolumn{2}{|c|}{ Tempo de prática na ATI (Anos) } \\
\hline 6 meses -1 & $69(17,4)$ \\
\hline $1-2$ & $73(18,4)$ \\
\hline$>2$ & $254(64,1)$ \\
\hline \multicolumn{2}{|c|}{ Frequência Semana (Dias na semana) } \\
\hline 1 & $02(0,5)$ \\
\hline 2 & $19(4,8)$ \\
\hline 3 & $68(17,2)$ \\
\hline$>3$ & $308(77,8)$ \\
\hline \multicolumn{2}{|c|}{ Prática de atividade física antes da ATI } \\
\hline Regularmente & $242(61,1)$ \\
\hline Às vezes & $17(4,3)$ \\
\hline Não & $137(34,6)$ \\
\hline \multicolumn{2}{|c|}{ Prática de atividade física além da realizada na ATI } \\
\hline Regularmente & $135(34,1)$ \\
\hline Às vezes & $11(2,8)$ \\
\hline Não & $250(63,1)$ \\
\hline \multicolumn{2}{|c|}{ Atividades praticadas } \\
\hline Ginástica & $120(30,3)$ \\
\hline Esporte & $16(4)$ \\
\hline Dança & $04(5,6)$ \\
\hline \multicolumn{2}{|c|}{ Depende de ajuda para ir a ATI } \\
\hline Sim & $02(0,5)$ \\
\hline Não & $394(99,5)$ \\
\hline \multicolumn{2}{|c|}{ Distância da ATI da residência } \\
\hline Muito longe & $05(1,2)$ \\
\hline Longe & $30(7,6)$ \\
\hline Perto & $260(65,7)$ \\
\hline Muito perto & $101(25,5)$ \\
\hline \multicolumn{2}{|c|}{ Idade de ingresso na ATI* (Anos) } \\
\hline $56-60$ & $88(22,2)$ \\
\hline $61-65$ & $99(25)$ \\
\hline $66-70$ & $76(19,2)$ \\
\hline $71-75$ & $51(12,9)$ \\
\hline $76-80$ & $48(12,1)$ \\
\hline
\end{tabular}


Continuação da Tabela 2

\begin{tabular}{lc}
\hline Variáveis & $\mathrm{n}(\%)$ \\
\hline Presença de doenças crônicas & $313(79)$ \\
Sim & $83(21)$ \\
Não & $216(55)$ \\
\hline Principais doenças crônicas** & $73(18)$ \\
Hipertensão & $59(15)$ \\
Artrose & \\
Diabetes & $346(87)$ \\
Percepção sobre a saúde após ingresso na ATI & $34(9)$ \\
Melhorou muito & $14(4)$ \\
Melhorou pouco & $00(0)$ \\
Sem diferença & \\
Piorou & \\
\hline
\end{tabular}

Fonte: $\mathrm{O}$ autor, 2017. n: número de sujeitos da amostra; ATI: Academia da Terceira Idade; *As faixas etárias inferiores e superiores às apresentadas obtiveram percentuais não expressivos; ${ }^{* *}$ As demais doenças citadas pelos investigados apresentaram percentuais não expressivos; Salário mínimo em 2017: R\$937,00.

A Tabela 3 apresenta os principais motivos que levaram os idosos a ingressar na ATI. Optou-se em mostrar somente os motivos que apresentaram mais que $10 \%$. Os dois principais fatores de motivação são relacionados à saúde, como "A necessidade de evitar problemas de saúde" e "O médico aconselhou praticar".
A Tabela 4 mostra os motivos que levam os idosos a permanecerem frequentando a ATI. Optou-se em relacionar somente os motivos que apresentaram um nível de acordo igual ou superior a 80\%. Destaca-se como tendo atingido $100 \%$ de acordo os motivos: "É uma atividade física saudável", "Gosto do/a meu/ minha professor/a" e "Me faz sentir bem".

Tabela 3. Motivos para o ingresso dos idosos frequentadores da ATI. $(\mathrm{n}=396$; Masculino $=58$; Feminino $=$ 338). Rio de Janeiro, RJ, 2017.

\begin{tabular}{llll}
\hline \multirow{2}{*}{ Motivos } & \multicolumn{3}{l}{ Ordem de importância } \\
\cline { 2 - 4 } & 1 & 2 & 3 \\
\cline { 2 - 4 } & $\mathrm{n}(\%)$ & $\mathrm{n}(\%)$ & $\mathrm{n}(\%)$ \\
\hline Sentir-se bem física e mentalmente & $51(13)$ & $55(14)$ & $71(18)$ \\
\hline Pela companhia de amigos e por novas amizades & $31(8)$ & $68(17)$ & $89(23)$ \\
\hline Para melhorar o condicionamento & $59(15)$ & $68(17)$ & $33(8)$ \\
\hline O médico aconselhou praticar & $65(16)$ & $17(4)$ & $22(6)$ \\
\hline Para evitar problemas de saúde & $101(26)$ & $82(21)$ & $44(11)$ \\
\hline
\end{tabular}

Fonte: O autor, 2017. 
Tabela 4. Motivos de permanência dos idosos nas ATIs. $(n=396$; Masculino= 58; Feminino= 338). Rio de Janeiro, RJ, 2017.

\begin{tabular}{|c|c|c|c|}
\hline \multirow[b]{2}{*}{ Motivos } & \multicolumn{3}{|c|}{ Nível de importância } \\
\hline & $\begin{array}{l}\text { Muito } \\
\text { importante } \\
\%\end{array}$ & $\begin{array}{l}\text { Mais ou menos } \\
\text { importante } \\
\%\end{array}$ & $\begin{array}{l}\text { Pouco } \\
\text { importante } \\
\%\end{array}$ \\
\hline O espaço é agradável & 90 & 10 & 0 \\
\hline As atividades são bem organizadas & 93 & 7 & 0 \\
\hline Me proporciona desafios & 84 & 7 & 9 \\
\hline É uma atividade física saudável & 100 & 0 & 0 \\
\hline Adoro fazer ginástica & 87 & 10 & 2 \\
\hline Fico mais resistente, forte e ágil & 97 & 3 & 0 \\
\hline Utilizo recursos e materiais legais durante as aulas & 82 & 12 & 6 \\
\hline Previne doenças & 97 & 2 & 1 \\
\hline Previne problemas de coluna, cardiovasculares e respiratórios & 95 & 3 & 2 \\
\hline Gosto de fazer novas amizades & 96 & 3 & 1 \\
\hline Gosto do/a meu/minha professor/a & 100 & 0 & 0 \\
\hline Me dá muita disposição & 88 & 2 & 0 \\
\hline Me dá satisfação & 99 & 1 & 0 \\
\hline Me diverte & 99 & 1 & 0 \\
\hline Me faz sentir bem & 100 & 0 & 0 \\
\hline O horário da aula não interfere nos meus outros compromissos & 91 & 5 & 4 \\
\hline O local de prática é perto da minha casa & 92 & 7 & 1 \\
\hline O local de prática possui facilidades de acesso & 99 & 1 & 0 \\
\hline O/A professor/a está sempre criando e ensinando algo diferente & 92 & 6 & 2 \\
\hline Os meus amigos me incentivam & 90 & 4 & 6 \\
\hline Preenche meu tempo de forma prazerosa & 98 & 2 & 0 \\
\hline Sou muito elogiado/a por praticar essa atividade física & 90 & 6 & 4 \\
\hline Tem muita gente legal praticando & 99 & 1 & 0 \\
\hline
\end{tabular}

Fonte: $\mathrm{O}$ autor, 2017.

\section{DISCUSSÃO}

Ressalta-se que a AP 2.1 (Zona Sul) é a área com o maior Índice de Desenvolvimento Humano (IDH) e renda per capita do município ${ }^{8} \mathrm{e}$ a segunda com mais idosos frequentando esse programa, que é público e gratuito. Embora isso pareça incongruente, pois o IDH poderia indicar que os idosos dessa região poderiam pagar para praticar EF em locais privados, observase que a renda da maioria dos idosos entrevistados é igual ou inferior a três salários mínimos, indicando que há comunidades de baixa renda em todas as APs do município, incluindo as consideradas áreas nobres. Considera-se que a renda apresentada pelos investigados é muito baixa, principalmente se forem considerados os gastos que os mesmos possuem com moradia, saúde e alimentação ${ }^{15}$, o que justifica a necessidade de participar de programas gratuitos de EF. Diferentemente, a região 3.3 (Grande Tijuca) possui a menor renda per capita ${ }^{8}$, justificando ser a área com mais sujeitos participantes no programa. Esses dados demonstram que o projeto atende, em sua maioria, a idosos que realmente necessitam da gratuidade para aderirem a um programa de EF.

Embora haja pequenas diferenças percentuais entre as regiões, a maioria da amostra é constituída por indivíduos do sexo feminino. Esse quadro vai 
ao encontro do apresentado em diferentes estudos já realizados sobre adesão de idosos a programas de EF, como o de Gilette et al. ${ }^{16}$ e Lemos et al. ${ }^{17}$. Goggin e Morrow Junior ${ }^{18}$ comentam que há uma maior probabilidade de as mulheres fazerem parte de programas de exercícios supervisionados por conta de tenderem a valorizar mais o trabalho em grupo. Em relação à baixa participação de homens, Mello et al. ${ }^{19}$ afirmam que estes tendem a considerar a ginástica como sendo uma atividade tipicamente feminina e, em decorrência desse estereótipo, sentem vergonha de atuar junto às mulheres por acharem que isso lhes trará prejuízos à reputação. Algo interessante comentado por Santos et al. ${ }^{20}$ é que muitas vezes os homens que ingressam em grupos de atividades direcionadas a sua faixa etária o fazem por influência de suas esposas e parentes. Sendo assim, é possível que idosos do sexo masculino que residem sozinhos sejam menos propensos a aderir a esse tipo de programa. Outro fator que pode explicar a presença majoritária de mulheres em programas como o investigado é o fenômeno de feminização da velhice ${ }^{21}$, que se dá pela diferença em relação à expectativa de vida entre os sexos. No Brasil, em média, as mulheres vivem cerca de sete anos a mais do que os homens ${ }^{22}$.

Em relação à idade dos participantes, as faixas etárias de maior adesão ao projeto são as mais baixas, tanto de ingressantes quanto dos que permanecem praticando, que reduzem a cada incremento da idade. A prevalência do ingresso no programa entre os 56 e 70 anos pode ser explicado pela idade para a aposentadoria no Brasil, que em 2014 era de 60 anos para os homens e 55 para as mulheres ${ }^{23}$. Uma vez aposentados, os idosos passam a ter mais tempo para dedicarem-se a outras atividades como, por exemplo, a prática regular de $\mathrm{EF}^{17}$, o que é positivo já que essa prática pode minimizar as perdas progressivas nos aspectos biopsicossociais advindas do processo de envelhecimento ${ }^{3,7}$.

Quanto à baixa taxa de ingresso entre os mais velhos, é possível que esteja relacionada ao processo natural de envelhecimento, que leva a alterações físicas, psicológicas e sociais e ao aumento no surgimento de doenças crônico-degenerativas ${ }^{24}$. Cabe ressaltar que tais alterações e doenças não são necessariamente um impeditivo para a prática regular e planejada de EF, que comprovadamente traria benefícios para combatê-las ${ }^{3}$. No entanto, pode estar prevalecendo a lógica de quanto mais avançada for a idade, maiores serão as chances de o indivíduo estar com a saúde comprometida e de ter a capacidade física reduzida, fatores que dificultam potencialmente a busca por programas de EF por parte dos idosos incluídos em faixas etárias avançadas, bem como a sua permanência nestes ${ }^{7,24}$. Outra explicação para esses resultados é o menor número de pessoas vivas nas faixas etárias mais elevadas. Assim, o menor número de praticantes é proporcional ao menor número de idosos existentes nas mesmas ${ }^{1}$.

Quanto ao tempo e à frequência semanal dedicados à prática de EF, verifica-se que a maioria dos idosos possui mais de dois anos de participação nas ATIs, com frequência predominante de mais de três dias semanais. De acordo com Prochaska e Velicer $^{25}$, só se pode considerar que um indivíduo aderiu a determinado comportamento uma vez que o pratique com consistência por um tempo mínimo de seis meses, visto que as etapas de mudança do comportamento citadas por esses autores levam aproximadamente esse tempo para transcorrerem, sendo essas etapas a pré-contemplação, contemplação, preparação, ação, manutenção e término.

Quanto ao tempo de prática semanal, considerando que cada aula tem duração de uma hora, indica um tempo de três horas ou mais de prática por semana, o que atende às recomendações Piercy et al. ${ }^{6}$. Portanto, pode-se inferir sobre a existência de importante adesão ao programa proposto nas ATIs da cidade do Rio de Janeiro.

Outro aspecto interessante levantado no presente estudo diz respeito ao nível de autonomia dos frequentadores das ATIs. A maioria dos idosos chega às praças a pé, condizendo com a porcentagem de idosos que considera importante que os locais de prática sejam perto e muito perto de suas casas. São poucos os idosos que recorrem ao transporte público ou particular para chegar às academias e os que o fazem normalmente estão frequentando, por algum motivo, uma praça em bairro diferente de onde moram. Esses dados corroboram os resultados de outros estudos, como o de Lopes et al. ${ }^{7}$ e Nakamura et al. ${ }^{26}$, que indicam que a proximidade do local de 
prática dos EF influencia na adesão, o que mostra a importância das ATIs estarem espalhadas por bairros estratégicos da cidade.

Apenas duas pessoas relataram necessitar de ajuda para chegar às respectivas ATIs. Esse dado, somado ao fato de que a maioria dos idosos vai a pé para o local de prática, indica que os frequentadores do programa mantêm autonomia funcional. Como a maioria dos idosos já frequenta as ATIs há dois anos ou mais, é possível inferir que o programa vem contribuindo para a manutenção dessa autonomia. Os bons resultados do trabalho desenvolvido nesses espaços também podem ser reforçados ao considerar que muitos idosos abandonaram os locais de prática de EF frequentados antes de ingressarem nas ATIs, para dedicarem-se exclusivamente a esse programa. Entretanto, é importante perceber que pode haver uma parcela da população que não está sendo servida adequadamente no que concerne ao acesso a programas de EF, em função da dificuldade de acessibilidade da cidade, devido as condições de manutenção das calçadas, a falta de rampas para facilitar a locomoção, a precariedade da segurança pública, entre outros aspectos.

Em relação à cultura físico-esportiva, verificouse que a maioria dos idosos antes de ingressarem nas ATIs praticava algum tipo de atividade física. Com esse dado é possível traçar um paralelo entre a prática pregressa e a adesão ao projeto. Machado et al. ${ }^{27}$ afirmam que as pessoas tenderão a escolher na velhice um estilo de vida semelhante aos já vivenciados na juventude e meia idade, incluindo a prática regular de EF. Nossos resultados corroboram tal afirmação, uma vez que há uma maior procura pelas ATIs entre idosos com uma cultura físicoesportiva consolidada. Em relação à prática atual, a maioria relata não praticar outra atividade além da oferecida pelas ATIs, o que pode indicar que os idosos entendem que suas necessidades são supridas por esse programa, embora não seja possível descartar a dificuldade financeira para custear paralelamente outra atividade. Independentemente do motivo, esse dado ressalta a importância dessas academias para a população idosa da cidade do Rio de Janeiro.

Os idosos que praticam alguma atividade física além das realizadas nas ATIs possuem como principal opção alguma modalidade que se enquadre na categoria de ginástica. Como a maioria da amostra é do sexo feminino, era esperado esse quadro, já que outros estudos sobre gênero e prática de EF de lazer já apontaram essa preferência entre as mulheres, a exemplo da investigação realizada por Silva et al. ${ }^{28}$.

A adesão, objeto central do presente estudo, é considerada o ingresso inicial e a permanência do indivíduo por um período de tempo superior a seis meses, em uma dada atividade ${ }^{13,14}$. No presente estudo, os motivos de ingresso e permanência nas ATIs foram levantados separadamente.

Uma forma de analisar a motivação que justifica o ingresso dos indivíduos a programas de EF é por meio dos valores atribuídos por estes à tal conduta. No caso, de acordo com Lovisolo ${ }^{29}$, as ações humanas decorrem de três motivos: de uma norma, da utilidade que a ação traz para o indivíduo, e/ou pelo gosto/ prazer que a ação provoca. Segundo o autor, no caso da norma, a ação executada decorre de uma regra, de uma imposição ou de uma convenção social. Já em relação à utilidade, a ação é desempenhada visando alcançar uma finalidade ou prestígio específicos. Ambos os casos caracterizam-se por motivações extrínsecas. No caso do gosto a ação é motivada e realizada fundamentalmente pela percepção de prazer que a atividade em si provoca no sujeito (motivações intrínsecas). Esses três motivos podem convergir ou divergir na execução de uma dada atividade, assim como podem estar presentes ao mesmo tempo. Optamos por utilizar essa perspectiva de análise para discutir os motivos de ingresso destacados pelos idosos entrevistados.

Para ingressarem nas ATIs, os idosos apresentaram prioritariamente motivos relacionados à prevenção e ou tratamento de problemas de saúde, indicando fortemente que a conduta desses sujeitos está sendo motivada pela utilidade que o EF pode desempenhar sobre sua saúde. Cabe destacar que 16\% dos sujeitos indicaram como fator mais importante o conselho médico, fator relacionado com uma conduta guiada pela norma. Esse resultado corrobora os achados da revisão sistemática desenvolvida por Harris et al. ${ }^{2}$, onde foi verificado que os motivos relacionados à saúde tendem a ser os principais determinantes para o ingresso de idosos em programas de EF. Não se 
pode negar a relevância desse resultado, pois ele indica que os idosos possuem grande preocupação com sua saúde, uma vez que já é consenso na literatura e amplamente divulgado que a prática regular de EF pode contribuir para uma vida mais saudável na velhice ${ }^{5}$. Entretanto, conduzir a opção por essa prática somente por motivos relacionados à utilidade e norma pode não favorecer à adesão propriamente dita ${ }^{13,14}$.

Vale ressaltar que a possibilidade de estar na companhia dos amigos e de fazer novas amizades, motivos relacionados ao prazer e consequentemente

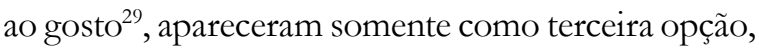
com 23\% da indicação dos idosos das ATIs. Do mesmo modo, o motivo "sentir-se bem física e mentalmente", relacionado em parte ao gosto, mas por outro lado também à utilidade, foi indicado por somente $18 \%$ dos sujeitos igualmente como terceira opção. Entretanto, embora indicados como terceira opção, esses motivos não podem ser desconsiderados no processo de adesão inicial de idosos à ATI, uma vez que a socialização é fundamental para a qualidade de vida da pessoa idosa ${ }^{30}$. Os demais motivos não receberam indicações expressivas.

Diferentemente dos motivos predominantes para justificar o ingresso, os fatores apontados pelos idosos como "muito importantes" para que permaneçam praticando as atividades nesses espaços, e que obtiveram 100\% de acordo entre os respondentes, estão em grande medida relacionados ao gosto dos indivíduos, a saber: "Gosto do meu/ minha professor/a" e "Me faz sentir bem". Figurando entre estes, foi encontrado somente um relacionado à utilidade dessa prática para a saúde, que é o motivo 'É uma atividade física saudável'. Ainda relacionados ao gosto, também figuram entre os motivos importantes os aspectos relacionados à satisfação, à diversão e ao bom relacionamento entre os praticantes: 'Me dá satisfação', 'Me diverte' e 'Tem muita gente legal praticando'. Esses motivos figuram com 99\% de acordo, e, não menos importante, o 'Gosto de fazer novas amizades', com um total de $96 \%$. Entre os motivos com 99\% de acordo ainda aparece 'O local de prática possui facilidades de acesso', relacionado à utilidade, o que reforça a importância da proximidade do espaço para a adesão de idosos aos $\mathrm{EF}^{26}$.
Portanto, diferentemente do que motiva as pessoas idosas a ingressarem em programas de EF, aspectos relacionados à motivação intrínseca, ao prazer em realizá-los, passa a ter um papel primordial na manutenção da conduta dos indivíduos, o que também foi constatado nos estudos inseridos e analisados na revisão sistemática de Harris et al. ${ }^{2}$, bem como em estudos desenvolvidos por Castro ${ }^{13,14}$ com diferentes populações. Esse dado é importante, pois indica que o programa precisa ter qualidade para estimular a permanência de seus usuários. O desempenho do(a) professor(a) no sentido da estimulação e da criação de estratégias de integração entre os praticantes parece ser elemento central para a adesão dos idosos, além da facilidade de acesso aos locais de aulas.

Ressalta-se ainda que o motivo "Tenho que me recuperar de uma lesão/doença", relacionado à utilidade dos EF voltados para a saúde, foi indicado pela maioria dos idosos como "pouco importante", indicando que estes indivíduos não procuram as ATIs para auxiliar na recuperação de lesões ou doenças agudas, ao contrário do que ocorre em relação às doenças crônicas, já que os idosos afirmaram ser esse um aspecto "muito importante" do trabalho desenvolvido nas ATIs.

Verificou-se que a doença com maior incidência no grupo investigado é a hipertensão arterial, o que era esperado, uma vez que, de acordo com a Sociedade Brasileira de Cardiologia, a hipertensão é uma doença com alta prevalência entre os idosos, e um dos maiores geradores de custos para a saúde pública no Brasil ${ }^{31}$. Destaca-se também a quantidade de pessoas com diabetes e artroses, doenças igualmente prevalentes na faixa etária investigada ${ }^{32,33}$.

Outros dados importantes são os relacionados às modificações percebidas pelos idosos em sua saúde desde o ingresso no projeto. A maioria considera que sua saúde melhorou muito após iniciarem os EF nas ATIs. Nenhum idoso relatou piora e apenas $4 \%$ do total considerou que não havia mudança nos fatores investigados. Esse resultado sugere que o trabalho desenvolvido nessas academias é importante para os cuidados com a saúde dos participantes e, possivelmente, leve à redução de gastos na atenção 
primária à saúde e quiçá na secundária, visto que a prática regular de EF é uma forma de controlar um dos maiores fatores de risco para doenças crônicas, que é o sedentarismo, além de ser uma das medidas aconselhadas no tratamento não medicamentoso dessas doenças, como a hipertensão $0^{31} \mathrm{e} o$ diabetes ${ }^{33}$. Dessa maneira, um programa público de EF com acompanhamento de professores tem potencial para constituir-se em uma ferramenta valiosa no combate às doenças crônicas, ao menos para idosos, melhorando a saúde e a qualidade de vida dessa crescente parcela da população.

Por fim, embora não diminua a importância dos resultados encontrados, vale ressaltar que este estudo apresenta como limitação o fato de não ter sido aplicada uma análise de associação entre as variáveis investigadas, o que traria maior compreensão quanto a importância de cada uma sobre o fenômeno da adesão dos idosos às ATIs.

\section{CONCLUSÃO}

Com relação ao objeto central desta pesquisa, conclui-se que, embora os motivos ligados à saúde figurem como importantes para os idosos ingressarem nas ATIs, a permanência no programa depende do gosto destes pela atividade e pelo ambiente de prática. Portanto, gestores, professores e equipe técnica necessitam cuidar da qualidade das aulas, do ambiente físico e da interação social onde as atividades acontecem.

\section{REFERÊNCIAS}

1. Instituto Brasileiro de Geografia Estatística. Número de idosos cresce 18\% em 5 anos e ultrapassa 30 milhões em 2017 [Internet]. 2018. [acesso 09 abr. 2020]. Disponível em: https://agenciadenoticias.ibge. gov.br/agencia-noticias/2012-agencia-de-noticias/ noticias/20980-numero-de-idosos-cresce-18-em-5anos-e-ultrapassa-30-milhoes-em-2017

2. Harris ERA, Porto F, Resende HG, Silva NSL. Free physical education programs for the elderly: a systematic review on adherence and abandonment. J Phys Educ. 2020;31(3149):1-14.
Também foi possível constatar uma grande adesão ao mesmo, o que facilita com que o $\mathrm{EF}$ ali praticado influencie positivamente na saúde, na qualidade de vida e na percepção de melhoria desses fatores por parte dos seus usuários, indicando que as ATIs podem ser consideradas uma boa ferramenta para contribuir com a saúde da população.

Os resultados obtidos sugerem que a ATI é um exemplo de política pública bastante valioso para a sociedade, especialmente por ser um programa em que a maioria de seus frequentadores é constituída por idosos de baixa renda, que possuem dificuldades de acesso a programas privados.

Assim sendo, este estudo possui aplicação prática potencial, pois oferece informações que podem contribuir para o planejamento e reformulações do projeto investigado, bem como para outros similares, que favoreçam a adesão de pessoas idosas à prática regular de EF.

Em que pese a relevância desses resultados, chama-se a atenção para uma lacuna importante, que é a falta de informação sobre a desistência de idosos a programas como o investigado. Sugere-se que estudos futuros explorem mais essa questão, visto que os motivos por trás da desistência também devem ser considerados em avaliações e possíveis reestruturações de projetos dessa natureza, a fim de contribuir para a redução das taxas de evasão.

Editado por: Ana Carolina Lima Cavaletti

3. Fragala MS, Cadore EL, Dorgo S, Izquierdo M, Kraemer WJ, Peterson MD, et al. Resistance training for older adults: Position Statement From the National Strength and Conditioning Association. J Strength Cond Res. 2019;33(8):2019-52.

4. Prefeitura do Rio de Janeiro [Internet]. Rio de Janeiro; 2018. Academias da Terceira Idade voltam a funcionar normalmente após recesso; [acesso 09 abr. 2020]. Disponível em: http://www.rio.rj.gov.br/web/guest/ exibeconteudo?id $=7614031$ 
5. Cordeiro RG, Monteiro W, Cunha F, Pescatello LS, Farinatti P. Influence of acute concurrent exercise performed in public fitness facilities on ambulatory blood pressure among older adults in Rio de Janeiro city. J Strength Cond Res. 2018;32(10):2962-70.

6. Piercy KL, Troiano RP, Ballard RM, Carlson SA, Fulton JE, Galuska DA, et al. The Physical Activity Guidelines for Americans. JAMA. 2018;320(19):2020-8.

7. Lopes MA, Krug RR, Bonetti A, Mazo GZ. Barreiras que influenciaram a não-adoção de atividade física por longevas. Rev Bras Ciênc Esporte. 2016;38(1):76-83.

8. Rio de Janeiro. Prefeitura. Anexo técnico I: informações sobre todas as áreas de planejamento [Internet]. Rio de Janeiro: Prefeitura;[201--?] [acesso 24 jul. 2020]. Disponível em: http://www.rio.rj.gov. br/dlstatic/10112/1529762/DLFE-220205.pdf/1.0

9. Netquest [Internet]. [S.1.]: Netquest; 2017. Calculadora de Amostras; [acesso 09 nov. 2018]. Disponível em: https://www.netquest.com/pt-br/home/paineisonline-pesquisas

10. Random.org [Internet]. Dublin: Rondomness and Integrity Service; 2016. List Randomizer; [acesso 12 out. 2016]. Disponível em: https://www.random. org/lists/

11. Barrow HM, McGee R. Barrow and McGee's practical measurement and assessment. $5^{\mathrm{a}}$ ed. Philadelphia: Lippincott Williams and Wilkins; 2000.

12. Conselho Nacional de Saúde. Resolução No 580, de 22 de março de 2018. Regulamentar o disposto no item XIII.4 da Resolução CNS no 466, de 12 de dezembro de 2012, que estabelece que as especificidades éticas das pesquisas de interesse estratégico para o Sistema Único de Saúde (SUS) serão contempladas em Resolução específica, e dá outras providências. Disponível em: https://conselho. saude.gov.br/resolucoes/2018/Reso580.pdf

13. Castro MS, Silva NL, Monteiro W, Palma A, Resende HG. Motivos de permanência dos praticantes nos programas de exercícios físicos oferecidos pelo Serviço Social do Comércio - Brasil. Motricidade. 2010;6(4):23-33.

14. Castro MS, Miranda M, Silva NL, Palma A, Resende HG. Motivos de ingresso nos programas de exercícios físicos oferecidos pelo Serviço Social do Comércio SESC-DF. Movimento. 2009;15(2):87-102.

15. Alvarenga LN, Kiyan L, Bitencourt B, Wanderley KS. Repercussões da aposentadoria na vida do idoso. Rev Esc Enferm USP. 2009;43(4):796-802.
16. Gillette DB, Petrescu-Prahova M, Herting JR, Belza B. A pilot study of determinants of ongoing participation in enhancefitness: a community-based group exercise program for older Adults. J Geriatr Phys Ther. 2015;38(4):194-201.

17. Lemos EC, Gouveia GC, Luna CF, Silva GB. Programa academia da cidade: descrição de fatores de adesão e não adesão. Rev Bras Ciênc Mov. 2016;24(4):75-84.

18. Goggin NL, Morrow JR. Physical activity behaviors of older adults. J Aging Phys Act. 2001;9:58-66.

19. Melo GF, Silva AA, Durães G, Cardoso FL, Formiga NS, Sousa IRC, et al. Estereótipos de gênero aplicados a homens atletas praticantes de esportes culturalmente femininos: a percepção de leigos, profissionais da Educação Física e atletas profissionais. Rev Bras Ciênc Mov .2015;23(3):30-7.

20. Santos PM, Marinho A, Mazo GZ, Benedetti TRB, Freitas CR. Lazer e participação de homens em grupos de convivência para idosos em Florianópolis (SC): motivações e significados. Rev Kairós. 2015;18(3):173-91.

21. Maximiano-Barreto MA, Portes FA, Andrade L, Campos LB, Generoso FK. A feminização da velhice: uma abordagem biopsicossocial do fenômeno. Interfaces Cient Hum Soc .2019;8(2):239-52.

22. Instituto Brasileiro de Geografia Estatística [Internet]. Rio de Janeiro: IBGE; [1995]- . Expectativa de vida dos brasileiros aumenta para 76,3 anos em 2018; 28 nov. 2019 [acesso 09 abr. 2020]. Disponível em: https://.ibge.gov.br/agencianoticias/2012-agencia-de-noticias/noticias/26103expectativa-de-vida-dos-brasileiros-aumenta-para76-3-anos-em-2018agenciadenoticias

23. Brasil. Ministério do Trabalho e Previdência Social, Instituto Nacional de Previdência Social, Empresa de Tecnologia e Informações da Previdência Social. Anuário Estatístico da Previdência Social/Ministério do Trabalho e Previdência Social - APES [Internet]. Brasília, DF: DATAPREV; 2014 [acesso 24 jul. 2020]. Disponível em: consultaesichttp://www..cgu.gov.br/ busca/dados/Lists/Pedido/Attachments/509652/ PEDIDO_AEPS-2014.pdf

24. Maresova P, Javanmardi E, Barakovic S, Husic BJ, Tomsone S, Krejcar O, et al. Consequences of chronic diseases and other limitations associated with old age: a scoping review. BMC Public Health. 2019;19(1):6-17.

25. Prochaska JO, Velicer WF. The Transtheoretical model of health behavior change. In: Glanz K, Rimer BK, Wiswanath K. Health Behavior: theory, research, and practice. São Francisco: Jossey-bass; 2015. 
26. Nakamura PM, Teixeira IP, Hino AAF, Kerr J, Kokubun. Association between private and public places and practice of physical activity in adults. Rev Bras Cineantropom Hum. 2016;18(3):297-310.

27. Machado RR, Azambuja CR, Pandolfo KCM, Santos DL. Nível de atividade física atual de idosas: influência do histórico de vida pregressa. Estud Interdiscipl Envelhec .2015;20(1):87-102.

28. Silva AA, Cardoso FL, Brandão AC, Ribeiro HL, Formiga NS, Melo GF. Cultura de gênero das modalidades de ginástica de academia do Brasil. Rev Bras Psicol Esporte. 2018;8(1):79-87.

29. Lovisolo H. Normas, utilidades e gostos na aprendizagem. In: Votre SJ, Castro, VLM, orgs. Cultura, atividade corporal e esporte. Rio de Janeiro: Editoria Central da Universidade Gama Filho; 1995.
30. Pereira MCA, Santos LFS, Moura TNB, Pereira LCA, Landim MBP. Contribuições da socialização e das políticas públicas para a promoção do envelhecimento saudável: uma revisão de literatura. Rev Bras Promoç Saúde. 2016;29(1):124-31.

31. Sociedade Brasileira de Cardiologia. $7^{\mathrm{a}}$ Diretriz brasileira de hipertensão arterial. Arq Bras Cardiol. 2016;107(3 Supl 3):1-82.

32. Dresch FK, Barcelos ARG, Cunha GL, Santos GA. Condição de saúde auto percebida e prevalência de doenças crônicas não transmissíveis em idosos atendidos pela estratégia da saúde da família. Conhecimento Online. 2017;9(2):118-27.

33. Reis CCB, Confortin C, Martins CG, Lodi FB, Roncada C, Rodrigues AD. Prevalência da prática de atividade física e correlação com a saúde de idosos de uma cidade no sul do Brasil. Rev Bras Reabilit Ativ Fís. 2016;5(1):27-32. 